Research Article

\title{
Asian Option Pricing under an Uncertain Volatility Model
}

\author{
Yuecai Han (iD) and Chunyang Liu (iD) \\ School of Mathematics, Jilin University, Changchun 130012, China \\ Correspondence should be addressed to Yuecai Han; hanyc@jlu.edu.cn and Chunyang Liu; liucy17@mails.jlu.edu.cn
}

Received 23 January 2020; Accepted 5 March 2020; Published 21 April 2020

Guest Editor: Zhimin Zhang

Copyright (C) 2020 Yuecai Han and Chunyang Liu. This is an open access article distributed under the Creative Commons Attribution License, which permits unrestricted use, distribution, and reproduction in any medium, provided the original work is properly cited.

In this paper, we study the asymptotic behavior of Asian option prices in the worst-case scenario under an uncertain volatility model. We derive a procedure to approximate Asian option prices with a small volatility interval. By imposing additional conditions on the boundary condition and splitting the obtained Black-Scholes-Barenblatt equation into two Black-Scholes-like equations, we obtain an approximation method to solve a fully nonlinear PDE.

\section{Introduction}

An option on a traded account is a financial contract that allows the buyer of the contract the right to trade an underlying asset for a specified price, called the strike price, during the lifetime of the option. There are various options, such as European options, American options, Asian options and barrier options. The foundation for the modern analysis of options, the Black-Scholes-Merton pricing formula for European options, was introduced by Black and Scholes [1] and Merton [2]. The Black-Scholes-Merton model assumes constant volatility. However, constant volatility cannot explain the observed market prices for options.

After Black, Scholes and Merton's work, some scholars studied option pricing models with stochastic volatility. A series of papers introduced several models for stochastic volatility, such as the Hull-White stochastic volatility model [3] and the Heston stochastic volatility model [4].

The uncertain volatility model is another approach to describe nonconstant volatility. In 1995, Lyons [5] and Avellaneda et al. [6] introduced uncertain volatility models. In these models, volatility is assumed to lie within a range of values, so prices are no longer unique. We can only get the best-case and worst-case scenario prices. Several studies investigate problems with uncertain volatility. We can see these results in Lyons [5], Avellaneda et al. [6], Dokuchaev and Savkin [7], Zhou and Li [8], and Forsyth and Vetzal [9].
These papers show pricing in uncertain volatility models involving nonlinear partial differential equations. Vorbrink [10] and Epstein and Ji [11] generalized the no-arbitrage theory to financial markets with ambiguous volatility in the mathematically rigorous framework of G-Brownian motion. Method of approximating the valuation equations and the latest research on Fourier transform was given by Zhang et al. [12] and Yu et al. [13]. Pooley et al. [14] and Avellaneda et al. [6] propose some numerical methods.

In 2014, Fouque and Ren [15] studied the price of European derivatives in the worst-case scenario with the uncertain volatility model. They provide an approximate method of pricing the derivatives with a small volatility interval. In addition, the paper also shows that the solution reduces to a constant volatility problem for simple options with convex payoffs.

This study examines the pricing problem of Asian options. The payoff function is path dependent on risky asset price processes with the addition of another variable to solve the problem. The first problem in estimating the worst-case scenario Asian option prices is obtaining the Hamilton-Jacobi-Bellman (HJB) equation for the prices. The HJB equation is called the Black-Scholes-Barenblatt (BSB) equation in financial mathematics. We can obtain the BSB equation using stochastic control theory. The next difficulty is to prove the convergence of the estimation. To control the error term, we obtain its expectation form using the 
Dynkin's formula and determine the conditions to impose on the payoff function through proof and deduction. Finally, we obtain the approximation procedure for the prices. Compared to Fouque and Ren's paper [15], we add an equation to the stochastic control system, which we can also reflect in the BSB equation. In terms of the dynamics of the risky asset price process, we provide an equation to describe the path dependence. When estimating the expectation form, we use the relationship between the two processes, in Section 4.4, we fix one of the two variables first to simplify the problem. We manage the two variables using another method that changes the form of the BSB equation.

The paper is organized as follows. In Section 2, we briefly describe Asian options under the uncertain volatility model and give the BSB equations for the option prices. In Section 3, we estimate the Asian option prices in the worst-case scenario, where the estimation relies on two Black-Scholeslike PDEs. Next, we propose the main result of this study, which shows the rationality of the estimation. In Section 4, we give the proof of the main result. Through the conditions imposed on the payoff function, we obtain the convergence of the error term. In the process, we obtain the expectation form of the error term, which we divide into three parts. We derive the control for each part using stochastic control theory and the properties of the worst-case scenario Asian option price process. Finally, we conclude the paper.

\section{Asian Options under Uncertain Volatility Model}

In this section, we introduce Asian options under the uncertain volatility model. Then, we provide the BSB equation of Asian option prices. Suppose that $\mathscr{X}$ is an Asian option written on a risky asset with maturity $T$ and payoff $\varphi(\cdot) \cdot \varphi(\cdot)$ is a nonconvex function and the result is identical to the Black-Scholes result under convex conditions. That is to say, this study results cover generalized Asian options. Here, generality means that the payoff function $\varphi(\cdot)$ can be in different forms, as long as it is nonconvex. Assume that the price process of the risky asset $X_{t}$ solves the stochastic differential equation:

$$
\mathrm{d} X_{t}=r X_{t} \mathrm{~d} t+\sigma_{t} X_{t} \mathrm{~d} W_{t},
$$

where $r$ is the constant risk-free interest rate and $W_{t}$ is a standard Brownian motion on the probability space $(\Omega, \mathfrak{F}, \mathbb{P})$. Let $\underline{\sigma}$ and $\bar{\sigma}$ are two constants and there is $\underline{\sigma} \leq \bar{\sigma}$. The volatility process $\sigma_{t} \in \mathscr{A}[\underline{\sigma}, \bar{\sigma}]$ for each $t \in[0, T]$, which is a family of progressively measurable and $[\underline{\sigma}, \bar{\sigma}]$-valued processes. By the abovementioned definition, we know that volatility in an uncertain volatility model is not a stochastic process with a probability distribution, but a family of stochastic processes with unknown prior information. Thus, we can use model ambiguity to distinguish between uncertain volatility models.

Due to the path dependence of risky asset price processes, we assume that $Y_{t, T}$ satisfies

$$
Y_{t, T}=\frac{Y_{T}-Y_{t}}{T-t}
$$

where $Y_{t}=\int_{0}^{t} X_{u} \mathrm{~d} u$. Then, we can obtain Asian option prices in the worst-case scenario at time $t<T$ as follows:

$$
V\left(t, X_{t}, Y_{t}\right)=\exp (-r(T-t)) \operatorname{esssup}_{\sigma \in \mathscr{A}[\underline{\sigma}, \bar{\sigma}]} E\left[\varphi\left(Y_{0, T}\right) \mid \mathfrak{\Im}_{t}\right]
$$

where esssup is the essential supremum. By the ambiguity of the uncertain volatility model, we obtain the definition of price as equation (3). Obviously, the worst-case scenario price is for the option seller and is related to the coherent risk measure that quantifies the model risk induced by volatility uncertainty (see [16]). Moreover, model ambiguity in mathematical finance has captured the attention of many. Therefore, we should pay attention to the importance of the worst-case prices.

Through stochastic control theory (see [17]), $V\left(t, X_{t}, Y_{t}\right)$ satisfies the HJB (BSB) equation.

Lemma 1. $V\left(t, X_{t}, Y_{t}\right)$ satisfies the following $B S B$ equation:

$$
\left\{\begin{array}{l}
\partial_{t} V+r\left(x \partial_{x} V-V\right)+x \partial_{y} V+\sup _{\sigma \in \mathscr{A}[\underline{\sigma}, \bar{\sigma}]}\left[\frac{1}{2} x^{2} \sigma^{2} \partial_{x x}^{2} V\right]=0, \\
0 \leq t \leq T, x \geq 0, y \geq 0, \\
V(T, x, y)=\varphi\left(\frac{y}{T}\right), \quad x \geq 0, y \geq 0 .
\end{array}\right.
$$

Proof. Note that the stochastic control system is

$$
\left\{\begin{array}{l}
\mathrm{d} X_{t}=r X_{t} \mathrm{~d} t+\sigma_{t} X_{t} \mathrm{~d} W_{t}, \quad \sigma_{t} \in \mathscr{A}[\underline{\sigma}, \bar{\sigma}], \\
\mathrm{d} Y_{t}=X_{t} \mathrm{~d} t .
\end{array}\right.
$$

Then, for all $(s, x, y) \in[0, T] \times R^{+} \times R^{+}$, we first establish the dynamic program frame:

$$
\left\{\begin{array}{l}
\mathrm{d} X_{t}=r X_{t} \mathrm{~d} t+\sigma_{t} X_{t} \mathrm{~d} W_{t} \\
\mathrm{~d} Y_{t}=X_{t} \mathrm{~d} t \\
X_{s}=x \\
Y_{s}=y
\end{array}\right.
$$

The cost function is

$$
J(s, x, y ; \sigma)=E_{s}\left[e^{-r(T-s)} \varphi\left(Y_{0, T}\right)\right],
$$

where $E_{s}[\cdot]=E\left[\cdot \mid \mathfrak{F}_{s}\right]$. The value function is

$$
V(s, x, y)=\operatorname{esssup}_{\sigma \in \mathscr{A}[\underline{\sigma}, \bar{\sigma}]} J(s, x, y ; \sigma) .
$$

For all $0 \leq s \leq \widehat{s} \leq T, \sigma \in \mathscr{A}[\underline{\sigma}, \bar{\sigma}]$, we have

$$
\begin{aligned}
V(s, x, y) & \geq E_{s}\left[e^{-r(T-s)} \varphi\left(Y_{0, T}\right)\right] \\
& =E_{s}\left[\int_{s}^{\widehat{s}}-r e^{-r(T-t)} \varphi \mathrm{d} t+e^{-r(T-\widehat{s})} \varphi\right] .
\end{aligned}
$$

Then, we obtain 


$$
0 \geq E_{s}\left[\int_{s}^{\widehat{s}}-r e^{-r(T-t)} \varphi \mathrm{d} t\right]+V(\widehat{s}, x, y)-V(s, x, y) .
$$

Dividing both sides of the inequality by $\widehat{s}-s$, we have

$$
0 \geq E_{s}\left[\frac{\int_{s}^{\widehat{s}}-r e^{-r(T-t)} \varphi \mathrm{d} t}{\widehat{s}-s}\right]+\frac{V(\widehat{s}, x, y)-V(s, x, y)}{\widehat{s}-s} .
$$

Here, we assume that $\varphi$ is Lipschitz continuous. Then, according to It $\widehat{o}$ 's formula and equation (6), we obtain

$$
\begin{aligned}
\mathrm{d} V= & V_{t} \mathrm{~d} t+V_{x} \mathrm{~d} X_{t}+V_{y} \mathrm{~d} Y_{t}+\frac{1}{2} V_{x x} \mathrm{~d} X_{t} \mathrm{~d} X_{t}+\frac{1}{2} V_{y y} \mathrm{~d} Y_{t} \mathrm{~d} Y_{t} \\
& +\frac{1}{2} V_{x y} \mathrm{~d} X_{t} \mathrm{~d} Y_{t} \\
= & \left(V_{t}+r X_{t} V_{x}+X_{t} V_{y}+\frac{1}{2} \sigma_{t}^{2} X_{t}^{2} V_{x x}\right) \mathrm{d} t+\sigma_{t} X_{t} V_{x} \mathrm{~d} W_{t} .
\end{aligned}
$$

Let $\widehat{s} \longrightarrow s$. For all $\sigma \in \mathscr{A}[\underline{\sigma}, \bar{\sigma}]$, we have

$$
\begin{aligned}
0 \geq & -r E_{s}\left[e^{-r(T-s)} \varphi\right]+V_{t}+r X_{s} V_{x}+X_{s} V_{y}+\frac{1}{2} \sigma_{s}^{2} X_{s}^{2} V_{x x} \\
\geq & -r V(s, x, y)+V_{t}(s, x, y)+r x V_{x}(s, x, y)+x V_{y}(s, x, y) \\
& +\frac{1}{2} \sigma_{s}^{2} X_{s}^{2} V_{x x}(s, x, y),
\end{aligned}
$$

which is

$$
0 \geq-r V+V_{t}+r x V_{x}+x V_{y}+\sup _{\sigma \in \mathscr{A}[\underline{\sigma}, \bar{\sigma}]} \frac{1}{2} \sigma^{2} x^{2} V_{x x} .
$$
that

In contrast, for any $\varepsilon>0$, there is a $\sigma(\varepsilon) \in \mathscr{A}[\underline{\sigma}, \bar{\sigma}]$ such

$$
\begin{aligned}
V(s, x, y)-\varepsilon(\widehat{s}-s) & \leq E_{s}\left[e^{-r(T-s)} \varphi\right] \\
& =E_{s}\left[\int_{s}^{\widehat{s}}-r e^{-r(T-t)} \varphi \mathrm{d} t\right]+E_{s}\left[e^{-r(T-\widehat{s})} \varphi\right] .
\end{aligned}
$$

Thus, we have

$$
-\varepsilon \leq E_{s}\left[\frac{\int_{s}^{\widehat{s}}-r e^{-r(T-t)} \varphi \mathrm{d} t}{\widehat{s}-s}\right]+\frac{V(\widehat{s}, x, y)-V(s, x, y)}{\widehat{s}-s} .
$$

From the argument above, we obtain

$$
0 \leq-r V+V_{t}+r x V_{x}+x V_{y}+\sup _{\sigma \in \mathscr{A}[\underline{\sigma}, \bar{\sigma}]} \frac{1}{2} \sigma^{2} x^{2} V_{x x} .
$$

Combining (14) with (17), we have

$$
0=-r V+V_{t}+r x V_{x}+x V_{y}+\sup _{\sigma \in \mathscr{A}[\underline{\sigma}, \bar{\sigma}]} \frac{1}{2} \sigma^{2} x^{2} V_{x x} .
$$

Remark 1 . Here, adding variable $Y$ into the dynamic system leads to a more complex stochastic control system, which adds the dimensionality of the BSB equation.

Remark 2. Note that (4) is a fully nonlinear PDE which has no solution, unlike the Black-Scholes equation. Thus, we solve the problem by reducing it to two Black-Scholes-like PDEs.

\section{Black-Scholes-Like PDEs and Main Result}

In this section, we first reparameterize the uncertain volatility model to study prices in the worst-case scenario. Assume that the risky asset price process satisfies the following SDE:

$$
\left\{\begin{array}{l}
\mathrm{d} X_{t}^{\varepsilon}=r X_{t}^{\varepsilon} \mathrm{d} t+\sigma_{t} X_{t}^{\varepsilon} \mathrm{d} W_{t} \\
\mathrm{~d} Y_{t}^{\varepsilon}=X_{t}^{\varepsilon} \mathrm{d} t
\end{array}\right.
$$

where $\quad \sigma_{t} \in \mathscr{A}^{\varepsilon}=\left\{\sigma_{t} \mid \sigma_{t}\right.$ is a $\quad\left[\sigma_{0}, \sigma_{0}+\varepsilon\right]-$ valued progressively measurable process $\}$ and $\sigma_{0} \in[\underline{\sigma}, \bar{\sigma}]$. The cost function is

$$
J^{\varepsilon}(t, x, y ; \sigma)=e^{-r(T-t)} E_{t x y}\left[\varphi\left(Y_{0, T}^{\varepsilon}\right)\right],
$$

where $E_{t x y}[\cdot]$ refers to the conditional expectation taken with respect to $X_{t}^{\varepsilon}=x, Y_{t}^{\varepsilon}=y$. The value function is

$$
V^{\varepsilon}(t, x, y ; \sigma)=\underset{\sigma \in \mathscr{A}^{\varepsilon}}{\operatorname{esssup}}\left[J^{\varepsilon}(t, x, y ; \sigma)\right] .
$$
$V^{\varepsilon}$

By Lemma 1, we obtain the following BSB equation for

$$
\left\{\begin{array}{l}
\partial_{t} V^{\varepsilon}+r\left(x \partial_{x} V^{\varepsilon}-V^{\varepsilon}\right)+x \partial_{y} V^{\varepsilon}+\sup _{\sigma \in \mathscr{A}^{\ell}} \frac{1}{2} x^{2} \sigma^{2} \partial_{x x}^{2} V^{\varepsilon}=0, \\
0 \leq t \leq T, x \geq 0, y \geq 0, \\
V^{\varepsilon}(T, x, y)=\varphi\left(\frac{y}{T}\right), \quad x \geq 0, y \geq 0,
\end{array}\right.
$$

which is equivalent to

$$
\left\{\begin{array}{l}
\partial_{t} V^{\varepsilon}+r\left(x \partial_{x} V^{\varepsilon}-V^{\varepsilon}\right)+x \partial_{y} V^{\varepsilon} \\
\quad+\sup _{\gamma \in \mathscr{A}[0,1]} \frac{1}{2} x^{2}\left(\sigma_{0}+\varepsilon \gamma\right)^{2} \partial_{x x}^{2} V^{\varepsilon}=0, \\
0 \leq t \leq T, x \geq 0, y \geq 0, \\
V^{\varepsilon}(T, x, y)=\varphi\left(\frac{y}{T}\right), \quad x \geq 0, y \geq 0,
\end{array}\right.
$$

where $\mathscr{A}[0,1]=\left\{\gamma_{t} \mid \gamma_{t}\right.$ is a valued progressively measurable process $\}$. It is obvious that 
the worst-case scenario price is higher than any Black- Scholes price with a constant volatility of $\sigma_{0} \in[\underline{\sigma}, \bar{\sigma}]$. In the following section, we will show that the worst-case scenario price of Asian options will converge to its Black-Scholes price with constant volatility $\sigma_{0}$. In addition, we can obtain the rate of convergence of the Asian option prices as the volatility interval shrinks to a single point. Then, we can estimate prices through this result when the interval is sufficiently small.

Let $V_{0}$ be the Black-Scholes prices, $V^{0}=\left.V^{\varepsilon}\right|_{\varepsilon=0}$, $V_{1}=\left.\partial_{\varepsilon} V^{\varepsilon}\right|_{\varepsilon=0}$. Now, we suppose that $V^{\varepsilon}$ is continuous with respect to $\varepsilon$. Then, by the continuity of $V^{\varepsilon}$ and equation (3), we have $V_{0}=V^{0}=\left.V^{\varepsilon}\right|_{\varepsilon=0}$. It is well known that $V_{0}$ satisfies the following partial differential equation:

$$
\left\{\begin{array}{l}
\partial_{t} V_{0}+r\left(x \partial_{x} V_{0}-V_{0}\right)+x \partial_{y} V_{0}+\frac{1}{2} \sigma_{0}^{2} x^{2} \partial_{x x}^{2} V_{0}=0 \\
0 \leq t \leq T, x \geq 0, y \geq 0 \\
V_{0}(T, x, y)=\varphi\left(\frac{y}{T}\right), \quad x \geq 0, y \geq 0
\end{array}\right.
$$

In contrast, we have $V_{1}=\left.\partial_{\varepsilon} V^{\varepsilon}\right|_{\varepsilon=0}$, which is the rate of convergence of the Asian option prices as $\varepsilon$ approaches 0 . To obtain the equation characterizing $V_{1}$, we differentiate both sides of equation (23) with respect to $\varepsilon$ and let $\varepsilon=0$, then we have

$$
\left\{\begin{array}{l}
\partial_{t} V_{1}+r\left(x \partial_{x} V_{1}-V_{1}\right)+x \partial_{y} V_{1}+\frac{1}{2} \sigma_{0}^{2} x^{2} \partial_{x x}^{2} V_{1} \\
\quad+\sup _{\gamma \in \mathscr{A}[0,1]} \gamma \sigma_{0} x^{2} \partial_{x x}^{2} V_{0}=0 \\
0 \leq t \leq T, x \geq 0, y \geq 0 \\
V_{1}(T, x, y)=0, \quad x \geq 0, y \geq 0
\end{array}\right.
$$

We now have two Black-Scholes-like PDEs. Next, we want to find the connection between $V^{\varepsilon}$ and $V_{0}, V_{1}$. Then, we try to prove whether it is possible to impose additional conditions on the payoff function to make the error term $V^{\varepsilon}-\left(V_{0}+\varepsilon V_{1}\right)$ be of order $o(\varepsilon)$. That is to say, the estimation of the worst-case scenario Asian option prices will approach the truth-value as the model ambiguity decreases. This will also provide a method to estimate the worst-case Asian option prices. By the deduction in Section 4, we obtain the following theorem, which is the main result of this study.

Theorem 1. Assume that $\varphi \in \mathscr{C}_{p}^{2}\left(R^{+}\right)$is Lipschitz continuous, the fourth derivative of $\varphi$ exists and the second derivative of $\varphi$ is continuous. Then,

$$
\lim _{\varepsilon \downarrow 0} \frac{V^{\varepsilon}-\left(V_{0}+\varepsilon V_{1}\right)}{\varepsilon}=0 .
$$

Here, $\varphi \in \mathscr{C}_{p}^{2}\left(R^{+}\right)$means that its derivatives up to order 2 have polynomial growth.
Remark 3. There are some difficulties in proving Theorem 1. The first is how to convert the error term into an estimable form. Here, we obtain its expectation form and divide it into three parts in Section 4. The second difficulty is how to estimate the three parts. Here, we will use stochastic control theory, the zero set property of equation (33), the properties of sublinear expectation in [18], and the properties of the worst-case scenario Asian option price processes.

Remark 4. By Theorem 1, we can compute Asian option price $V^{\varepsilon}\left(t, X_{t}^{\varepsilon}, Y_{t}^{\varepsilon}\right) \quad$ with its approximation, $V_{0}\left(t, X_{t}^{\varepsilon}, Y_{t}^{\varepsilon}\right)+\varepsilon V_{1}\left(t, X_{t}^{\varepsilon}, Y_{t}^{\varepsilon}\right)$, where $V_{0}\left(t, X_{t}^{\varepsilon}, Y_{t}^{\varepsilon}\right)$ is the Black-Scholes price of the Asian option and we can compute $V_{1}\left(t, X_{t}^{\varepsilon}, Y_{t}^{\varepsilon}\right)$ numerically by a simple difference scheme according to (25) (see [14]).

Remark 5. Note that (24) and (25) are independent of $\varepsilon$. Thus, when we compute $V^{\varepsilon}$ with different $\varepsilon$, we only need to compute $V_{0}$ and $V_{1}$ once for all small values of $\varepsilon$ by Theorem 1.

\section{Proof of the Main Result}

In this section, we try to control the error term to prove that we can compute $V^{\varepsilon}$ with its estimation $V_{0}+\varepsilon V_{1}$. Additionally, from the conditions imposed on $\varphi$ mentioned in Theorem 1, we have the following process of proof. The following parts also reflect our thought process.

4.1. The Lipschitz Continuity of the Payoff Function. From Section 3, we know that only with the continuity of $V^{\varepsilon}$ can we obtain the PDEs of $V_{0}=\left(\left.V^{\varepsilon}\right|_{\varepsilon=0}\right)$ and $V_{1}=\left(\left.\partial_{\varepsilon} V^{\varepsilon}\right|_{\varepsilon=0}\right)$. Thus, to obtain the continuity of $V^{\varepsilon}$, we suppose that $\varphi$ is Lipschitz continuous. Then, there exists a constant $K_{1}$ such that

$$
|\varphi(x)-\varphi(y)| \leq K_{1}|x-y|, \quad \text { for all } x \neq y, x, y \in R^{+} .
$$

Thus, we have the following Lemma.

Lemma 2. Assume that $\varphi$ is Lipschitz continuous. Then, $V^{\varepsilon}$ is continuous with respect to $\varepsilon$.

Proof. Let $0 \leq \varepsilon_{0} \leq \varepsilon<1$. Note that

$$
V^{\varepsilon}(t, x, y ; \sigma)=\underset{\sigma \in \mathscr{A}^{\varepsilon}}{\operatorname{essup}}\left\{e^{-r(T-t)} E_{t x y}\left[\varphi\left(Y_{0, T}^{\mathcal{\varepsilon}}\right)\right]\right\} .
$$

We have

$$
\begin{aligned}
e^{r(T-t)} V^{\varepsilon_{0}}(t, x, y ; \sigma) & =\underset{\sigma \in \mathscr{A}^{\varepsilon_{0}}}{\operatorname{esssup}} E_{t x y}\left[\varphi\left(Y_{0, T}^{\varepsilon_{0}}(\sigma)\right)\right] \\
& =\underset{\sigma \in \mathscr{A}^{\varepsilon}}{\operatorname{esssup}} E_{t x y}\left[\varphi\left(Y_{0, T}^{\varepsilon}\left(\sigma \wedge\left(\sigma_{0}+\varepsilon_{0}\right)\right)\right)\right] .
\end{aligned}
$$

By the Lipschitz continuity of $\varphi$ and equation (1), there is a constant $K_{1}$ such that 


$$
\begin{aligned}
& e^{r(T-t)}\left|V^{\varepsilon}(t, x, y ; \sigma)-V^{\varepsilon_{0}}(t, x, y ; \sigma)\right| \\
& \quad \leq \underset{\sigma \in \mathscr{A}^{\varepsilon}}{\operatorname{esssup}}\left|E_{t x y}\left[\varphi\left(Y_{0, T}^{\varepsilon}(\sigma)\right)\right]-E_{t x y}\left[\varphi\left(Y_{0, T}^{\varepsilon}\left(\sigma \wedge\left(\sigma_{0}+\varepsilon_{0}\right)\right)\right)\right]\right| \\
& \quad \leq K_{1} \underset{\sigma \in \mathscr{A}^{\varepsilon}}{\operatorname{esssup}}\left(E_{t x y}\left|Y_{0, T}^{\varepsilon}(\sigma)-Y_{0, T}^{\varepsilon}\left(\sigma \wedge\left(\sigma_{0}+\varepsilon_{0}\right)\right)\right|^{2}\right)^{(1 / 2)} \\
& \quad \leq\left(K_{1} / T\right) \underset{\sigma \in \mathscr{A}^{\varepsilon}}{\operatorname{esssup}}\left(E_{t x y} \int_{0}^{T}\left|X_{u}^{\varepsilon}(\sigma)-X_{u}^{\varepsilon}\left(\sigma \wedge\left(\sigma_{0}+\varepsilon_{0}\right)\right)\right|^{2} \mathrm{~d} u\right)^{(1 / 2)} .
\end{aligned}
$$

With the estimates of the moments of solutions of the stochastic differential equations (Theorem 9 in Section 2.9 and Corollary 12 in Section 2.5 of [19]), we have the constants

$N=N\left(q, r, \sigma_{0}\right), N^{\prime}=N^{\prime}\left(q, r, \sigma_{0}\right)$, and $C=\max \left\{N N^{\prime}, N+\right.$ $N\}^{\prime}$ such that

$$
\begin{aligned}
& E_{t x y}\left[\sup _{s \leq u}\left|X_{s}^{\varepsilon}(\sigma)-X_{s}^{\varepsilon}\left(\sigma \wedge\left(\sigma_{0}+\varepsilon_{0}\right)\right)\right|^{2 q}\right] \\
& \leq N u^{q-1} e^{N u} E_{t x y}\left[\int_{0}^{u}\left|X_{s}^{\varepsilon}(\sigma)\right|^{2 q} \cdot\left|\sigma_{s}-\sigma_{s} \wedge\left(\sigma_{s}+\varepsilon_{0}\right)\right|^{2 q} \mathrm{~d} s\right] \\
& \leq N u^{q-1} e^{N u} N^{\prime} e^{N^{\prime} u} u\left(1+x^{2 q}\right)\left|\varepsilon-\varepsilon_{0}\right|^{2 q} \\
& \quad=C u^{q} e^{C u}\left(1+x^{2 q}\right)\left|\varepsilon-\varepsilon_{0}\right|^{2 q} .
\end{aligned}
$$

Thus, we have

$$
\begin{aligned}
& e^{r(T-t)}\left|V^{\varepsilon}(t, x, y)-V^{\varepsilon_{0}}(t, x, y)\right| \\
& \leq \frac{K_{1}}{T} \underset{\sigma \in \mathscr{A}^{\varepsilon}}{\operatorname{esssup}}\left(\int_{0}^{T} E_{t x y} \sup _{s \in[0, u]}\left|X_{u}^{\varepsilon}(\sigma)-X_{u}^{\varepsilon}\left(\sigma \wedge\left(\sigma_{0}+\varepsilon_{0}\right)\right)\right|^{2} \mathrm{~d} u\right)^{(1 / 2)} \\
& \quad \leq \frac{K_{1}}{T} \operatorname{esssup}_{\sigma \in \mathscr{A}^{\varepsilon}}\left(\int_{0}^{T} C u e^{C u}\left(1+x^{2}\right)\left|\varepsilon-\varepsilon_{0}\right|^{2} \mathrm{~d} u\right)^{(1 / 2)} \\
& \leq K_{1}^{\prime}\left(1+x^{2}\right)^{(1 / 2)}\left|\varepsilon-\varepsilon_{0}\right|
\end{aligned}
$$

where $K_{1}^{\prime}=K_{1}^{\prime}\left(K_{1}, C, T\right)$.

Let $\varepsilon \longrightarrow \varepsilon_{0}$. We have $\left|V^{\varepsilon}(t, x, y)-V^{\varepsilon_{0}}(t, x, y)\right| \longrightarrow 0$.

The continuity of $V^{\varepsilon}$ with respect to $\varepsilon$ can be proven similarly when $\varepsilon \leq \varepsilon_{0}$.

4.2. Expectation Form of the Error Term. In this section, we analyze the error term and give its expectation form as preparation work before proving the convergence of $V_{0}+\varepsilon V_{1}$

Let $\widehat{\sigma}_{t}$ be the worst-case scenario volatility process and $\widehat{X}_{t}^{\varepsilon}$ be the worst-case scenario risky asset process. Then, we can rewrite equation (19) as follows:

$$
\left\{\begin{array}{l}
\mathrm{d} \widehat{X}_{t}^{\varepsilon}=r \widehat{X}_{t}^{\varepsilon} \mathrm{d} t+\widehat{\sigma}_{t} \widehat{X}_{t}^{\varepsilon} \mathrm{d} W_{t}, \\
\mathrm{~d} \widehat{Y}_{t}^{\varepsilon}=\widehat{X}_{t}^{\varepsilon} \mathrm{d} t .
\end{array}\right.
$$

We can obtain the expression of $\widehat{\sigma}$ by equation (23), and we have $\widehat{\sigma}(\varepsilon)=\sigma_{0}+\varepsilon \widehat{\gamma}$, where

$$
\widehat{\gamma}(t, x, y ; \varepsilon)= \begin{cases}1, & \partial_{x x}^{2} V^{\varepsilon}(t, x, y) \geq 0, \\ 0, & \partial_{x x}^{2} V^{\varepsilon}(t, x, y)<0 .\end{cases}
$$

Similarly, by solving equation (25) for $V_{1}$, we have the volatility process, $\bar{\sigma}(\varepsilon)=\sigma_{0}+\varepsilon \bar{\gamma}$, where

$$
\bar{\gamma}(t, x, y)= \begin{cases}1, & \partial_{x x}^{2} V_{0}(t, x, y) \geq 0, \\ 0, & \partial_{x x}^{2} V_{0}(t, x, y)<0 .\end{cases}
$$

Here, we use the short notation $\widehat{\gamma}_{t}$ and $\bar{\gamma}_{t}$ for $\widehat{\gamma}(t, x, y ; \varepsilon)$ and $\bar{\gamma}(t, x, y)$, respectively. Let $Z^{\varepsilon}=V^{\varepsilon}-\left(V_{0}+\varepsilon V_{1}\right)$. To estimate the error term $Z^{\varepsilon}$, we define the operator $L(\sigma)=\partial_{t}+r x \partial_{x}-r+(1 / 2) \sigma^{2} x^{2} \partial_{x x}^{2}+x \partial_{y}$. According to partial differential equations (22), (24) and (25), we have

$$
\begin{aligned}
L\left(\widehat{\sigma}_{t}\right) Z^{\varepsilon}= & L\left(\widehat{\sigma}_{t}\right)\left(V^{\varepsilon}-\left(V_{0}+\varepsilon V_{1}\right)\right) \\
= & 0-L\left(\widehat{\sigma}_{t}\right)\left(V_{0}+\varepsilon V_{1}\right) \\
= & -\left(L\left(\widehat{\sigma}_{t}\right)-L\left(\sigma_{0}\right)\right) V_{0}-L\left(\sigma_{0}\right) V_{0}-\varepsilon\left(L\left(\widehat{\sigma}_{t}\right)\right. \\
& \left.-L\left(\sigma_{0}\right)\right) V_{1}-\varepsilon L\left(\sigma_{0}\right) V_{1} \\
= & \varepsilon\left(\bar{\gamma}_{t}-\widehat{\gamma}_{t}\right) \sigma_{0} x^{2} \partial_{x x}^{2} V_{0}-\frac{\varepsilon^{2}}{2}\left(\left(\widehat{\gamma}_{t}\right)^{2} x^{2} \partial_{x x}^{2} V_{0}\right. \\
& \left.+2 \sigma_{0} \widehat{\gamma}_{t} x^{2} \partial_{x x}^{2} V_{1}\right)-\frac{\varepsilon^{3}}{2}\left(\widehat{\gamma}_{t}\right)^{2} x^{2} \partial_{x x}^{2} V_{1} \\
= & -f^{\varepsilon}(t, x, y),
\end{aligned}
$$

with the boundary condition $Z^{\varepsilon}(T)=V^{\varepsilon}(T)-V_{0}$ $(T)-\varepsilon V_{1}(T)=0$. We have the following expectation form of $Z^{\varepsilon}$ by the Dynkin's formula:

$$
\begin{aligned}
Z^{\varepsilon}= & E_{t x y}\left[\int_{t}^{T} f^{\varepsilon}(s, x, y) \mathrm{d} s\right] \\
= & \varepsilon E_{t x y}\left[\int_{t}^{T}\left(\widehat{\gamma}_{s}-\bar{\gamma}_{s}\right) \cdot \sigma_{0} \cdot\left(\widehat{X}_{s}^{\varepsilon}\right)^{2} \partial_{x x}^{2} V_{0}\left(s, \widehat{X}_{s}^{\varepsilon}, \widehat{Y}_{s}^{\varepsilon}\right) \mathrm{d} s\right] \\
& +\varepsilon^{2} E_{t x y}\left[\int _ { t } ^ { T } \left\{\frac{1}{2}\left(\widehat{\gamma}_{s}\right)^{2}\left(\widehat{X}_{s}^{\varepsilon}\right)^{2} \partial_{x x}^{2} V_{0}\left(s, \widehat{X}_{s}^{\varepsilon}, \widehat{Y}_{s}^{\varepsilon}\right)\right.\right. \\
& \left.\left.+\sigma_{0}\left(\widehat{\gamma}_{s}\right)\left(\widehat{X}_{s}^{\varepsilon}\right)^{2} \wp_{x x}^{2} V_{1}\left(s, \widehat{X}_{s}^{\varepsilon}, \widehat{Y}_{s}^{\varepsilon}\right)\right\} \mathrm{d} s\right] \\
& +\varepsilon^{3} E_{t x y}\left[\int_{t}^{T} \frac{1}{2}\left(\widehat{\gamma}_{s}\right)^{2}\left(\widehat{X}_{s}^{\varepsilon}\right)^{2} \partial_{x x}^{2} V_{1}\left(s, \widehat{X}_{s}^{\varepsilon}, \widehat{Y}_{s}^{\varepsilon}\right) \mathrm{d} s\right] \\
= & \varepsilon I_{1}+\varepsilon^{2} I_{2}+\varepsilon^{3} I_{3},
\end{aligned}
$$

where 


$$
\begin{aligned}
& I_{1}= E_{t x y}\left[\int_{t}^{T}\left(\widehat{\gamma}_{s}-\bar{\gamma}_{s}\right) \cdot \sigma_{0} \cdot\left(\hat{X}_{s}^{\varepsilon}\right)^{2} \partial_{x x}^{2} V_{0}\left(s, \widehat{X}_{s}^{\varepsilon}, \widehat{Y}_{s}^{\varepsilon}\right) \mathrm{d} s\right] \\
& I_{2}= E_{t x y}\left[\int _ { t } ^ { T } \left\{\frac{1}{2}\left(\widehat{\gamma}_{s}\right)^{2}\left(\widehat{X}_{s}^{\varepsilon}\right)^{2} \partial_{x x}^{2} V_{0}\left(s, \widehat{X}_{s}^{\varepsilon}, \widehat{Y}_{s}^{\varepsilon}\right)\right.\right. \\
&\left.\left.+\sigma_{0}\left(\widehat{\gamma}_{s}\right)\left(\widehat{X}_{s}^{\varepsilon}\right)^{2} \partial_{x x}^{2} V_{1}\left(s, \widehat{X}_{s}^{\varepsilon}, \widehat{Y}_{s}^{\varepsilon}\right)\right\} \mathrm{d} s\right] \\
& I_{3}= E_{t x y}\left[\int_{t}^{T} \frac{1}{2}\left(\widehat{\gamma}_{s}\right)^{2}\left(\hat{X}_{s}^{\varepsilon}\right)^{2} \partial_{x x}^{2} V_{1}\left(s, \hat{X}_{s}^{\varepsilon}, \widehat{Y}_{s}^{\varepsilon}\right) \mathrm{d} s\right] \\
&\left|Z^{\varepsilon}\right| \leq \varepsilon\left|I_{1}\right|+\varepsilon^{2}\left|I_{2}\right|+\varepsilon^{3}\left|I_{3}\right| .
\end{aligned}
$$
$\left|I_{3}\right|$.

We can therefore estimate $Z^{\varepsilon}$ by controlling $\left|I_{1}\right|,\left|I_{2}\right|$, and

\subsection{The Polynomial Growth Condition of the Payoff Function.} From Section 4.2, we know that to control the error term, we need to analyze the three parts. By (41), we have

$$
\left|\frac{Z^{\varepsilon}}{\varepsilon}\right| \leq\left|I_{1}\right|+\varepsilon\left(\left|I_{2}\right|+\varepsilon\left|I_{3}\right|\right) .
$$

Therefore, it is sufficient to prove

$$
\lim _{\varepsilon \downarrow 0}\left|I_{1}\right|+\varepsilon\left(\left|I_{2}\right|+\varepsilon\left|I_{3}\right|\right)=0 \text {. }
$$

Obviously, it is necessary to obtain controls of the terms $\left|I_{2}\right|$ and $\left|I_{3}\right|$. For $\left|I_{1}\right|$, we need to prove its convergence. We first consider controls of the terms $\left|I_{2}\right|$ and $\left|I_{3}\right|$.

By the expressions of $I_{2}$ and $I_{3}$, we can see that the partial derivatives of $V_{0}$ and $V_{1}$ are involved. Thus, we should consider estimating them before controlling $I_{2}$ and $I_{3}$. Next, we can obtain the expectation form of $V_{0}$ and $V^{\varepsilon}$ by the classical result. When $\varepsilon=0$, we have

$$
X(u)=x \exp \left\{\left(r-\frac{\sigma_{0}^{2}}{2}\right)(u-t)+\sigma_{0}\left(W_{u}-W_{t}\right)\right\} .
$$

Thus,

$$
\begin{aligned}
V_{0}(t, x, y) & =e^{-r(T-t)} E_{t x y}\left[\varphi\left(Y_{0, T}\right)\right] \\
& =e^{-r(T-t)} E_{t x y}\left[\varphi\left(\frac{1}{T} \int_{0}^{T} X(u) \mathrm{d} u\right)\right] \\
& =e^{-r(T-t)} E_{t x y}\left[\varphi\left(\frac{1}{T} \cdot x \cdot\left(\int_{0}^{T} e^{\left(r-\left(\sigma_{0}^{2} / 2\right)\right)(u-t)+\sigma_{0}\left(W_{u}-W_{t}\right)} \mathrm{d} u\right)\right)\right] \\
& =e^{-r(T-t)} E_{t x y}[\varphi(x \cdot H)],
\end{aligned}
$$

where $\quad H\left(=(1 / T) \int_{0}^{T} \exp \left\{\left(r-\left(\sigma_{0}^{2} / 2\right)\right)(u-t)+\sigma_{0}\left(W_{u^{-}}\right.\right.\right.$ $\left.\left.\left.W_{t}\right)\right\} \mathrm{d} u\right)$ is a random variable for fixed $t \in[0, T]$. Similarly, we have

$$
\begin{aligned}
V^{\varepsilon}(t, x, y) & =e^{-r(T-t)} \operatorname{esssup}_{\sigma \in \mathscr{A}^{\varepsilon}}\left\{E_{t x y}\left[\varphi\left(Y_{0, T}^{\varepsilon}\right)\right]\right\} \\
& =e^{-r(T-t)} E_{t x y}[\varphi(x \cdot G)],
\end{aligned}
$$

where $\quad G\left(=(1 / T) \int_{0}^{T} \exp \left\{\left(r-\left(\widehat{\sigma}_{u}\right)^{2} / 2\right)(u-t)-\widehat{\sigma}_{u}\left(W_{u}-\right.\right.\right.$ $\left.\left.\left.W_{t}\right)\right\} \mathrm{d} u\right)$ is a random variable for a fixed $t \in[0, T]$.

By equations (45) and (46), we note that it is necessary to impose polynomial growth conditions on $\varphi$ to control $\partial_{x x}^{2} V_{0}$ and $\partial_{x x}^{2} V^{\varepsilon}$. Then, we estimate $\partial_{x x}^{2} V_{0}(t, x, y)$ and $\partial_{x x}^{2} V^{\varepsilon}(t, x, y)$ in the following Lemma.

Lemma 3. Suppose that the second derivative of the payoff function satisfies the polynomial growth condition, that is, there are constants $K_{2}$ and $m$ such that $\varphi \prime \prime(x) \leq K_{2}\left(1+|x|^{m}\right)$. Then, we have constant $K_{3}$ such that

$$
\left|\partial_{x x}^{2} V_{0}(t, x, y)\right| \leq K_{3}\left(1+|x|^{m}\right),
$$

where $K_{3}$ depends on $T, t, E_{t x y}\left[|H|^{2}\right], E_{t x y}\left[|H|^{m+2}\right]$, and $K_{2}$. Moreover, there is a constant $K_{4}$ such that

$$
\left|\partial_{x x}^{2} V^{\varepsilon}(t, x, y)\right| \leq K_{4}\left(1+|x|^{m}\right)
$$

where $K_{4}$ depends on $T, t, E_{t x y}\left[|G|^{2}\right], E_{t x y}\left[|G|^{m+2}\right]$, and $K_{2}$.

Proof. As the assumption of $\varphi$ in the lemma, we have

$$
\begin{aligned}
\partial_{x x}^{2} V_{0}(t, x, y) & =e^{-r(T-t)} E_{t x y}\left[\varphi \prime \prime(x H) H^{2}\right] \\
& \leq e^{-r(T-t)} E_{t x y}\left[K_{2}\left(1+|x H|^{m}\right) H^{2}\right] \\
& \leq K_{3}\left(1+|x|^{m}\right) .
\end{aligned}
$$
$K_{2}$.

Here, $K_{3}$ depends on $T, t, E_{t x y}\left[|H|^{2}\right], E_{t x y}\left[|H|^{m+2}\right]$, and

Indeed, for a constant $m>0$, we have

$$
\begin{aligned}
E H^{m} & =\frac{1}{(T)} E\left(\int_{-t}^{T-t} \exp \left\{\left(\frac{r-\sigma_{0}^{2}}{2}\right) u+\sigma_{0} W_{u}\right\} \mathrm{d} u\right)^{m} \\
& \leq\left(\frac{1}{T}\right)^{m} E\left(\int_{-t}^{T-t} e^{\left|r-\sigma_{0}^{2} / 2\right|(T-t)+\sigma_{0} W_{u}} \mathrm{~d} u\right)^{m} \\
& \leq\left(\frac{1}{T}\right)^{m} e^{m\left|r-\sigma_{0}^{2} / 2\right|(T-t)} E\left(\sup _{s \in(-t, T-t)}\left\{e^{\sigma_{0} W_{s}}\right\}\right)^{m}<+\infty .
\end{aligned}
$$


We obtain the control tial ${ }_{x x}^{2} V^{\varepsilon}$ similarly. Then, there is a constant $K_{4}$ that depends on $T, t, E_{t x y}\left[|G|^{2}\right], E_{t x y}\left[|G|^{m+2}\right]$, and $K_{2}$ such that

$$
\left|\partial_{x x}^{2} V^{\varepsilon}(t, x, y)\right| \leq K_{4}\left(1+|x|^{m}\right) .
$$

Now, by the following proposition, we can obtain controls of the terms $\left|I_{2}\right|$ and $\left|I_{3}\right|$.

Proposition 1. Assume that $\varphi \in \mathscr{C}_{p}^{2}\left(R^{+}\right)$and the Lipschitz continuity condition holds. Then, there exist constants $C_{1}$ and $p_{1}$ such that $I_{2}$ and $I_{3}$ in equations (39) and (40) satisfy

$$
\left|I_{2}\right|+\left|I_{3}\right| \leq C_{1}\left(1+|x|^{p_{1}}\right) .
$$

Proof. By Lemma 3, we have the following inequality from (23) and (48):

$$
\begin{aligned}
\left|\partial_{t} V^{\varepsilon}+r\left(x \partial_{x} V^{\varepsilon}-V^{\varepsilon}\right)+x \partial_{y} V^{\varepsilon}\right| & \leq\left|\frac{1}{2}\left(\sigma_{0}+\varepsilon\right)^{2} x^{2} \partial_{x x}^{2} V^{\varepsilon}\right| \\
& \leq\left|\left(\frac{K_{4}}{2}\right)\left(\sigma_{0}+\varepsilon\right)^{2}\left(|x|^{2}+|x|^{m+2}\right)\right|
\end{aligned}
$$

By the expression of $V_{1}$, it is true that

$$
\left|\partial_{t} V_{1}+r\left(x \partial_{x} V_{1}-V_{1}\right)+x \partial_{y} V_{1}\right| \leq\left|K_{4} \sigma_{0}\left(|x|^{2}+|x|^{m+2}\right)\right| \text {. }
$$

By (25) and (47), we get the controls for $x^{2} \partial_{x x}^{2} V_{1}$ :

$$
\begin{aligned}
\left|x^{2} \partial_{x x}^{2} V_{1}\right| & =\left|\partial_{t} V_{1}+r\left(x \partial_{x} V_{1}-V_{1}\right)+x \partial_{y} V_{1}+\bar{g}_{t} \sigma_{0} x^{2} \partial_{x x}^{2} V_{0}\right| \cdot\left(\frac{2}{\sigma_{0}^{2}}\right) \\
& \leq\left(\left|\partial_{t} V_{1}+r\left(x \partial_{x} V_{1}-V_{1}\right)+x \partial_{y} V_{1}\right|+\left|\sigma_{0} x^{2} \partial_{x x}^{2} V_{0}\right|\right) \cdot\left(\frac{2}{\sigma_{0}^{2}}\right) \\
& \leq M_{1}\left(|x|^{2}+|x|^{m+2}\right),
\end{aligned}
$$

where $M_{1}$ depends on $K_{3}, K_{4}$, and $\sigma_{0}$. We can obtain the existence and uniqueness of $\widehat{X}_{t}^{\varepsilon}$ from Theorem 5.2.1 in [20]. Then, by the estimates of the moments of solutions of the stochastic differential equations (Corollary 12 in Section 2.5 of [19]), there is a constant $N_{1}(q)$ for a fixed $q>0$ such that

$$
E_{t x y}\left[\sup _{s \in[t, T]}\left|\hat{X}_{s}^{\varepsilon}\right|^{q}\right] \leq N_{1}(q) e^{N_{1}(q)(T-t)}\left(1+|x|^{q}\right) .
$$

By (40), (55), and (56), we have the following inequality:

$$
\begin{aligned}
\left|I_{3}\right| & =\left|E_{t x y}\left[\int_{t}^{T} \frac{1}{2}\left(\widehat{\gamma}_{s}\right)^{2}\left(\hat{X}_{s}^{\varepsilon}\right)^{2} \partial_{x x}^{2} V_{1}\left(s, \widehat{X}_{s}^{\varepsilon}, \widehat{Y}_{s}^{\varepsilon}\right) \mathrm{d} s\right]\right| \\
& \leq\left(\frac{M_{1}}{2}\right) E_{t x y}\left[\int_{t}^{T}\left(\left|\hat{X}_{s}^{\varepsilon}\right|^{2}+\left|\widehat{X}_{s}^{\varepsilon}\right|^{m+2}\right) \mathrm{d} s\right] \leq M_{1}^{\prime}\left(1+|x|^{m+2}\right) .
\end{aligned}
$$

Here, $M_{1}^{\prime}$ depends on $T, t, N_{1}(2), N_{1}(m+2)$, and $M_{1}$. By (39), (47), (55), and (56), we obtain the control of term $\left|I_{2}\right|$.

$$
\begin{aligned}
\left|I_{2}\right|= & \left|E_{t x y}\left[\int_{t}^{T} \frac{1}{2}\left(\widehat{\gamma}_{s}\right)^{2}\left(\widehat{X}_{s}^{\varepsilon}\right)^{2} \partial_{x x}^{2} V_{0}+\sigma_{0}\left(\widehat{\gamma}_{s}\right)\left(\widehat{X}_{s}^{\varepsilon}\right)^{2} \partial_{x x}^{2} V_{1} \mathrm{~d} s\right]\right| \\
\leq & \frac{K_{3}}{2} E_{t x y}\left[\int_{t}^{T}\left(\widehat{X}_{s}^{\varepsilon}\right)^{2}+\left(\widehat{X}_{s}^{\varepsilon}\right)^{m+2} \mathrm{~d} s\right] \\
& +M_{1} E_{t x y}\left[\int_{t}^{T}\left(\widehat{X}_{s}^{\varepsilon}\right)^{2}+\left(\widehat{X}_{s}^{\varepsilon}\right)^{m+2} \mathrm{~d} s\right] \\
\leq & M_{2}\left(1+|x|^{p_{1}}\right),
\end{aligned}
$$

where $M_{2}$ depends on $T, t, M_{1}, K_{3}, N_{1}(2)$, and $N_{1}(m+2)$, $p_{1} \geq m+2$.Combining (57) and (58), we have a constant $C_{1}$ such that

$$
\left|I_{2}\right|+\left|I_{3}\right| \leq C_{1}\left(1+|x|^{p_{1}}\right) .
$$

4.4. The Continuity of the Second Derivative of the Payoff Function. By Proposition 1, we obtain controls of the terms $\left|I_{2}\right|$ and $\left|I_{3}\right|$. Next, for the fixed point $(t, x, y) \in[0, T] \times R^{+} \times R^{+}$, it suffices to prove that

$$
\lim _{\varepsilon \downarrow 0}\left|I_{1}\right|=0 .
$$

Note that if $\varphi \in \mathscr{C}_{p}^{2}\left(R^{+}\right)$(i.e., its derivatives up to order 2 have polynomial growth), we can obtain the following inequality by (38), (47), (56), and Hölder inequality:

$$
\begin{aligned}
\left|I_{1}\right| \leq & {\left[E_{t x y}\left[\int_{t}^{T}\left(\sigma_{0}\left(\hat{X}_{s}^{\varepsilon}\right)^{2} \partial_{x x}^{2} V_{0}\right)^{2} \mathrm{~d} s\right]\right]^{(1 / 2)} } \\
& \cdot\left[E_{t x y}\left[\int_{t}^{T}\left(\hat{\gamma}_{s}-\bar{\gamma}_{s}\right)^{2} \mathrm{~d} s\right]\right]^{(1 / 2)} \\
\leq & M_{3}\left(1+|x|^{p_{2}}\right)^{(1 / 2)}\left[E_{t x y}\left[\int_{t}^{T}\left|\widehat{\gamma}_{s}-\bar{\gamma}_{s}\right| \mathrm{d} s\right]\right]^{(1 / 2)} .
\end{aligned}
$$

Here, $M_{3}$ depends on $K_{3}, T, t, \sigma_{0}$, and $p_{2} \geq 4+2 m$. Moreover, $M_{3}$ is independent of $\varepsilon$.

Let $h^{\varepsilon}(t, x, y)=\widehat{\gamma}(t, x, y ; \varepsilon)-\bar{\gamma}(t, x, y)$. By (34) and (35), we have

$$
\left|h^{\varepsilon}(t, x, y)\right|= \begin{cases}1, & \partial_{x x}^{2} V^{\varepsilon} \partial_{x x}^{2} V_{0}<0, \\ 0, & \partial_{x x}^{2} V^{\varepsilon} \partial_{x x}^{2} V_{0} \geq 0 .\end{cases}
$$
that

Thus, to prove $\left|I_{1}\right| \longrightarrow 0$ as $\varepsilon \longrightarrow 0$, it suffices to prove

$$
\lim _{\varepsilon \downarrow 0} E_{t x y}\left[\int_{t}^{T}\left|h^{\varepsilon}\left(s, \hat{X}_{s}^{\varepsilon}, \widehat{Y}_{s}^{\varepsilon}\right)\right| \mathrm{d} s\right]=0 .
$$

By the expression of $h^{\varepsilon}$, we should analyze the derivatives of $V_{0}$ and $V^{\varepsilon}$. Here, we find that the continuity of $\varphi^{\prime \prime}$ is necessary.

Lemma 4. Assume that $\varphi^{\prime \prime}$ is continuous. Then, $\partial_{x x}^{2} V_{0}$ and $\partial_{x x}^{2} V^{\varepsilon}$ are continuous with respect to $(x, y)$. 
Proof. By (45), we have $V_{0}(t, x, y)=e^{-r(T-t)} E_{t x y}[\varphi(x H)]$ and $\partial_{x x}^{2} V_{0}(t, x, y)=e^{-r(T-t)} E_{t x y}\left[\varphi^{\prime \prime}(x H) H^{2}\right]$. If $\varphi^{\prime \prime}$ is continuous, then for all $x_{0} \in R^{+}, \delta>0$, there is a constant $\xi=\xi\left(\delta, x_{0}\right)$ such that

$$
\left|\varphi^{\prime \prime}(x H)-\varphi^{\prime \prime}\left(x_{0} H\right)\right| \leq \delta
$$

for all $x H \in\left(x_{0} H-\xi, x_{0} H+\xi\right)$. So, for all $\left(x_{0}, y_{0}\right) \in R^{+} \times R^{+}, \quad x H \in\left(x_{0} H-\xi, x_{0} H+\xi\right), \quad$ and $y \in\left(y_{0}-\xi, y_{0}+\xi\right)$, we have

$$
\begin{aligned}
\left|\partial_{x x}^{2} V_{0}(t, x, y)-\partial_{x x}^{2} V_{0}\left(t, x_{0}, y_{0}\right)\right| & =e^{-r(T-t)}\left|E_{t x y}\left[\varphi^{\prime \prime}(x H) H^{2}-\varphi^{\prime \prime}\left(x_{0} H\right) H^{2}\right]\right| \\
& \leq e^{-r(T-t)} E_{t x y}\left[H^{2}\left|\varphi^{\prime \prime}(x H)-\varphi^{\prime \prime}\left(x_{0} H\right)\right|\right] \\
& \leq e^{-r(T-t)} \delta E_{t x y}\left[H^{2}\right] .
\end{aligned}
$$

Thus, we obtain

$$
\lim _{(x, y) \longrightarrow\left(x_{0}, y_{0}\right)} \partial_{x x}^{2} V_{0}(t, x, y)=\partial_{x x}^{2} V_{0}\left(t, x_{0}, y_{0}\right)
$$

Similarly, we can obtain the continuity of $\partial_{x x}^{2} V^{\varepsilon}$.

Remark 6. Rationally, $V^{\varepsilon}$ and its derivatives converge to $V_{0}$ and its corresponding derivatives as $\varepsilon$ approaches 0 by Lemma 2.

Remark 7. To simplify the complexity brought by the variable $Y$, which is called path dependence, and to study the behavior of $h^{\varepsilon}$, we define

$$
D_{t y}^{\lambda}=\left\{x \in R^{+} \mid \partial_{x x}^{2} V^{\varepsilon_{0}} \partial_{x x}^{2} V_{0} \leq 0, \exists \varepsilon_{0}>\lambda\right\} .
$$

Let $D_{t y}^{0}=\lim _{\lambda \downarrow 0} D_{t y}^{\lambda}$. Then, we can obtain the following equation when $\partial_{x x}^{2} V^{\varepsilon}$ is continuous:

$$
D_{t y}^{0}=\left\{x \in R^{+} \mid \partial_{x x}^{2} V_{0}(t, x, y)=0\right\} .
$$

Remark 8. To control $h^{\varepsilon}$, we divide $D_{t y}^{\lambda}$ into two parts. Let $\alpha(\rho)=[-\rho, \rho]$. We will discuss the characteristics of $D_{t y}^{\lambda} \cap \alpha(\rho)$ and $D_{t y}^{\lambda} \cap \alpha(\rho)^{c}$.

Lemma 5. Assume that $\varphi^{\prime \prime}$ is continuous and the fourth derivative of $\varphi$ exists. Then, we have

$$
P_{t x y}\left(D_{s y_{s}}^{0} \cap \alpha(\rho)\right)=0, \quad \text { for } s \in[t, T] .
$$

Here, $P_{t x y}(\cdot)$ refers to the conditional probability with respect to $X_{t}^{\varepsilon}=x$ and $Y_{t}^{\varepsilon}=y$.

Proof. By (33) and (24), we can obtain the equation

$$
\left\{\begin{array}{l}
2 \partial_{t} V_{0}+r\left(x \partial_{x} V_{0}-V_{0}\right)+\frac{1}{2} \sigma_{0}^{2} x^{2} \partial_{x x}^{2} V_{0}=0, \\
V_{0}(T)=\varphi(x H) .
\end{array}\right.
$$

Let $Q=\partial_{x x}^{2} V_{0}$. Then, by equation (70) and the existence of the fourth derivative of $\varphi$, we have

$$
\left\{\begin{array}{l}
2 \partial_{t} Q+\left(r+\sigma_{0}^{2}\right) Q+\left(r+2 \sigma_{0}^{2}\right) x \partial_{x} Q+\frac{1}{2} \sigma_{0}^{2} x^{2} \partial_{x x}^{2} Q=0, \\
Q(T)=\varphi^{\prime \prime}(x H) H^{2} .
\end{array}\right.
$$

Let $x=\log k$. Then, we have

$$
\left\{\begin{array}{l}
2 \partial_{t} Q+\left(r+\sigma_{0}^{2}\right) Q+\left(r+2 \sigma_{0}^{2}\right) \partial_{k} Q+\frac{1}{2} \sigma_{0}^{2} \partial_{k}^{2} Q=0, \\
Q(T)=\varphi^{\prime \prime}((\log k) H) H^{2} .
\end{array}\right.
$$

Note that the coefficients in equation (72) are constants and $Q$ is bounded on $D_{s y_{s}}^{0} \cap \alpha(\rho)$ by the continuity of $\varphi^{\prime \prime}$ and Lemma 4. Moreover, by equation (72), we find that $y$ has no relationship with the equations. Then, by Theorem A of [21] and the remark below it, we find that the number of zero points of $Q$ is only countable for all $\left(s, y_{s}\right) \in[t, T] \times R$. Thus, $\partial_{x x}^{2} V_{0}$ has only countable zero points. Hence, we have $P_{t x y}\left(D_{s y_{s}}^{0} \cap \alpha(\rho)\right)=0$ by Lemma 4.10 of [15] and then the proof of Lemma 5 is complete.

Based on the previous analysis, we will now prove (63). We split the expectation into two parts. By proving the convergence of each part, we can show the convergence of the expectation.

Proposition 2. Assume that $\varphi \in \mathscr{C}_{p}^{2}\left(R^{+}\right), \varphi^{\prime \prime}$ is continuous, and the fourth derivative of $\varphi$ exists. Then, we obtain equation (63).

Proof. Let $\bar{D}_{t y}^{\lambda}$ be the closure of $D_{t y}^{\lambda}, \bar{D}_{t y}^{0}=\lim _{\lambda \downarrow 0} \bar{D}_{t y}^{\lambda}$, and $0 \leq \lambda<\varepsilon<1$.

By the definition of $D_{t y}^{\lambda}$, we have 


$$
\begin{aligned}
& E_{t x y}\left[\int_{t}^{T}\left|h^{\varepsilon}\left(s, \widehat{X}_{s}^{\varepsilon}, \widehat{Y}_{s}^{\varepsilon}\right)\right| \mathrm{d} s\right] \\
& \leq E_{t x y}\left[\int_{t}^{T} \mathbb{Q}_{\left.\bar{D}_{(\widehat{x}}^{\lambda}\right)}\left(\hat{X}_{s}^{\varepsilon}\right) \mathrm{d} s\right] \\
& =E_{t x y}\left[\int_{t}^{T} \mathbb{Q}_{\bar{D}_{\left(\hat{s Y}_{s}\right)}^{\lambda} \cap \alpha(\rho)}\left(\hat{X}_{s}^{\varepsilon}\right) \mathrm{d} s\right] \\
& +E_{t x y}\left[\int_{t}^{T} \mathbb{Q}_{\bar{D}_{\left(s \hat{s}_{s}\right)}^{\lambda} \cap \alpha(\rho)^{c}}\left(\widehat{X}_{s}^{\varepsilon}\right) \mathrm{d} s\right] \\
& =\Phi_{1}+\Phi_{2} \text {. }
\end{aligned}
$$

Now, we consider the second part of (73) first. By (56) and Chebyshev's inequality,

$$
\begin{aligned}
\Phi_{2} & \leq E_{t x y}\left[\int_{t}^{T} \mathbb{1}_{\alpha(\rho)^{c}}\left(\widehat{X}_{s}^{\varepsilon}\right) \mathrm{d} s\right] \\
& \leq \int_{t}^{T} P_{t x y}\left(\sup _{s \in[t, T]}\left|\widehat{X}_{s}^{\varepsilon}\right| \geq \rho\right) \mathrm{d} s \\
& \leq \frac{T-t}{\rho} E_{t x y}\left[\sup _{s \in[t, T]}\left|\widehat{X}_{s}^{\varepsilon}\right|\right] \\
& \leq \frac{(T-t) N_{1}(1)}{\rho} e^{N_{1}(1)(T-t)}(1+|x|) .
\end{aligned}
$$

Thus, we have

$$
\lim _{\rho \longrightarrow \infty} \Phi_{2}=0 .
$$

For the first part, we note that

$$
\left.\Phi_{1}=\int_{t}^{T} P_{t x y}\left(\widehat{X}_{s}^{\varepsilon} \in \bar{D}_{\left(\widehat{Y}_{s}\right)}^{\lambda}\right) \cap \alpha(\rho)\right) \mathrm{d} s .
$$

Let $\theta(\Omega)=\sup _{\lambda \in[0,1]} P_{t x y}(\Omega)$; then,

$$
P_{t x y}\left(\widehat{X}_{s}^{\varepsilon} \in \bar{D}_{\left(s \hat{Y}_{s}^{\varepsilon}\right)}^{\lambda} \cap \alpha(\rho)\right) \leq \theta\left(\bar{D}_{\left(s \widehat{Y}_{s}^{\varepsilon}\right)}^{\lambda} \cap \alpha(\rho)\right) .
$$

Note that $\lambda<\varepsilon$. Then, $\bar{D}_{s y}^{\lambda}$ is a sequence of decreasing closed sets as $\varepsilon \downarrow 0$. Obviously, $\widehat{X}_{s}^{\varepsilon}$ converges weakly to $X_{s}$. Thus, $\left\{X_{s}\right\}$ is weakly compact. By Lemma 8 in [18], we can see that

$$
\lim _{\varepsilon \downarrow 0} \theta\left(\bar{D}_{\left(\widehat{s Y}_{s}\right)}^{\lambda} \cap \alpha(\rho)\right)=\theta\left(\bar{D}_{\left(\hat{s Y}_{s}^{0}\right)}^{0} \cap \alpha(\rho)\right) .
$$
have

By Lemma 4 , there is $\bar{D}_{s y}^{0}=D_{s y}^{0}$. Then, by Lemma 5 , we

$$
P_{t x y}\left(\widehat{X}_{s}^{\varepsilon} \in \bar{D}_{\left(s \hat{Y}_{s}^{\varepsilon}\right)}^{0} \cap \alpha(\rho)\right)=0, \quad \text { for } \varepsilon \geq 0 .
$$

Next, by the definition of $\theta(\Omega)$, we have

$$
\theta\left(\bar{D}_{\left(\hat{Y}_{s}^{0}\right)}^{0} \cap \alpha(\rho)\right)=0 .
$$

Thus,

$$
\lim _{\varepsilon \downarrow 0} \theta\left(\bar{D}_{\left(\widehat{s Y_{s}}\right)}^{\lambda} \cap \alpha(\rho)\right)=0 .
$$

Then, we obtain

$$
\lim _{\varepsilon \downarrow 0} \Phi_{1}=\lim _{\varepsilon \downarrow 0} P_{t x y}\left(\widehat{X}_{s}^{\varepsilon} \in \bar{D}_{\left(s \widehat{Y}_{s}^{\varepsilon}\right)}^{\lambda} \cap \alpha(\rho)\right)=0 .
$$

By equations (75) and (82), for any $\delta>0$, there is $\rho_{0}=$ $\rho_{0}(t, x, y, \delta)>0$ such that

$$
\Phi_{2}<\frac{\delta}{2}, \quad \text { for all } \rho>\rho_{0} .
$$

Next, for a given $\rho_{0}$ and $\delta$, there is $\varepsilon_{0}=\varepsilon_{0}\left(t, x, y, \delta, \rho_{0}(t, x, y, \delta)\right)$ such that

$$
\Phi_{1}<\frac{\delta}{2}, \quad \text { for all } \varepsilon<\varepsilon_{0} .
$$
that

Therefore, for any $\delta>0$, there is $\varepsilon_{0}=\varepsilon_{0}(t, x, y, \delta)$ such

$$
\Phi_{1}+\Phi_{2}<\delta, \quad \text { for all } \varepsilon<\varepsilon_{0}
$$

i.e.,

$$
\lim _{\varepsilon \downarrow 0} E_{t x y}\left[\int_{t}^{T}\left|h^{\varepsilon}\left(s, \widehat{X}_{s}^{\varepsilon}, \widehat{Y}_{s}^{\varepsilon}\right)\right| \mathrm{d} s\right]=0 .
$$

4.5. Proof of the Main Result. Now, from the analysis above, we can give the brief proof of Theorem 1.

By inequality (61) and Proposition 2, we have

$$
\lim _{\varepsilon \downarrow 0}\left|I_{1}\right|=0 .
$$

By inequality (41), we have

$$
\left|\frac{V^{\varepsilon}-\left(V_{0}+\varepsilon V_{1}\right)}{\varepsilon}\right| \leq\left|I_{1}\right|+\varepsilon\left(\left|I_{2}\right|+\varepsilon\left|I_{3}\right|\right)=0 .
$$

By Proposition 1 and equation (87), we obtain the theorem.

\section{Conclusion}

In this study, we analyze the behavior of Asian option prices in the worst-case scenario using an uncertain volatility model with volatility interval $\left[\sigma_{0}, \sigma_{0}+\varepsilon\right]$. As $\varepsilon$ approaches 0 , the ambiguity of the model vanishes. We can also see that the worst-case scenario prices of Asian options converge to its Black-Scholes prices with constant volatility as the interval shrinks. Additionally, this study provides an approach to estimate the worst-case scenario Asian option prices. At the same time, we also provide an estimation method to solve a fully nonlinear PDE (22) by imposing additional conditions on the boundary condition and splitting it into two Black-Scholes-like equations.

\section{Data Availability}

No data were used to support this study. 


\section{Conflicts of Interest}

The authors declare that they have no conflicts of interest.

\section{Acknowledgments}

This paper was partially supported by NSFC (Grant nos. 11871244 and 11901233).

\section{References}

[1] F. Black and M. Scholes, "The pricing of options and corporate liabilities," Journal of Political Economy, vol. 81, no. 3, pp. 637-654, 1973.

[2] R. C. Merton, "Theory of rational option pricing," The Bell Journal of Economics and Management Science, vol. 4, no. 1, pp. 141-183, 1973.

[3] J. Hull and A. White, "The pricing of options on assets with stochastic volatilities," The Journal of Finance, vol. 42, no. 2, pp. 281-300, 1987.

[4] S. L. Heston, "A closed-form solution for options with stochastic volatility with applications to bond and currency options," Review of Financial Studies, vol. 6, no. 2, pp. 327343, 1993.

[5] T. J. Lyons, "Uncertain volatility and the risk-free synthesis of derivatives," Applied Mathematical Finance, vol. 2, no. 2, pp. 117-133, 1995.

[6] M. Avellaneda, A. Levy, and A. ParÁS, "Pricing and hedging derivative securities in markets with uncertain volatilities," Applied Mathematical Finance, vol. 2, no. 2, pp. 73-88, 1995.

[7] N. G. Dokuchaev and A. V. Savkin, "The pricing of options in a financial market model with transaction costs and uncertain volatility," Journal of Multinational Financial Management, vol. 8, no. 2-3, pp. 353-364, 1998.

[8] Q. Zhou and X. Li, "Vulnerable options pricing under uncertain volatility model," Journal of Inequalities and Applications, vol. 2019, no. 1, p. 315, 2019.

[9] P. A. Forsyth and K. R. Vetzal, "Implicit solution of uncertain volatility/transaction cost option pricing models with discretely observed barriers," Applied Numerical Mathematics, vol. 36, no. 4, pp. 427-445, 2001.

[10] J. Vorbrink, "Financial markets with volatility uncertainty," Journal of Mathematical Economics, vol. 53, no. 8, pp. 64-78, 2014.

[11] L. G. Epstein and S. Ji, "Ambiguous volatility and asset pricing in continuous time," Review of Financial Studies, vol. 26, no. 7, pp. 1740-1786, 2013.

[12] Z. Zhang, Y. Yong, and W. Yu, "Valuing equity-linked death benefits in general exponential Lévy models," Journal of Computational and Applied Mathematics, vol. 365, Article ID 112377, 2020.

[13] W. Yu, Y. Yong, G. Guan, Y. Huang, W. Su, and C. Cui, "Valuing guaranteed minimum death benefits by cosine series expansion," Mathematics, vol. 7, no. 9, p. 835, 2019.

[14] D. M. Pooley, P. A. Forsyth, and K. R. Vetzal, "Numerical convergence properties of option pricing pdes with uncertain volatility," IMA Journal of Numerical Analysis, vol. 23, no. 2, pp. 241-267, 2003.

[15] J.-P. Fouque and B. Ren, "Approximation for option prices under uncertain volatility," SIAM Journal on Financial Mathematics, vol. 5, no. 1, pp. 360-383, 2014.

[16] R. Cont, "Model uncertainty and its impact on the pricing of derivative instruments," Mathematical Finance, vol. 16, no. 3, pp. 519-547, 2006.
[17] J. Yong and X. Zhou, Stochastic Controls, Hamiltonian Systems and HJB Equations, Springer, New York, NY, USA, 1999.

[18] L. Denis, M. Hu, and S. Peng, "Function spaces and capacity related to a sublinear expectation: application to G-Brownian motion paths," Potential Analysis, vol. 34, no. 2, pp. 139-161, 2011.

[19] N. Krylov, Controlled Diffusion Processes, Springer, Berlin, Germany, 1980.

[20] B. Øksendal, Stochastic Differential Equations, Springer, Berlin, Germany, 2003.

[21] S. Angenent, "The zero set of a solution of a parabolic equation," Journal für die Reine und Angewandte Mathematik, vol. 390, pp. 79-96, 1988. 